montrent, sans contestation possible, la supériorité du chien sur l'homme dans le travail de découverte. Cette supériorité défie toute comparaison, même pour les chiens dont les temps ont été les plus longs $\$ . .$.

Le Comité d'organisation des expériences faites à Nancy a formulé d'autre part les conclusions suivantes :

“ $1^{\circ} \mathrm{Il}$ ressort des résultats consignés... que la question de l'utilisation du chien pour la recherche des blessés est définitivement résolue.

$2^{c}$ Que sous bois ou sur des terrains très accidentés et surtout la nuit, le chien est capable de découvrir, en un temps très court, des blessés qui, ayant perdu connaissance... ne peuvent appeler à leur secours.

$3^{\circ}$ Qu'il est du plus haut intérêt de continuer ces expériences, en utilisant surtout des chiens de grande quête et à nez puissant; qu'il faut se placer, au cours du dressage, pour le choix du terrain et de la méthode d'exploration, dans les conditions se rapprochant le plus possible de celles du temps de guerre. »

Dr F.

\title{
LES VOITURES AUTOMOBILES DANS LE SERVICE DE SANTÉ
}

Celle question, d'intérêt primordial, aujourd'hui que l'automobilisme s'est adapté à tous les besoins de transport, est traitée d'une façon très complète, dans une série d'articles par $M$. le Dr Dommartin, médecin-major de $1^{\text {re }}$ classe parus dans le Caducée ${ }^{1}$.

Evidemment, aussi bien les services de transports automobiles sont appropriés aux besoins de l'armée, - et déjà plusieurs armées d'Europe sont pourvues d'un service auxiliaire d'automobilistes volontaires, - aussi bien ce mode de transport sera le bienvenu dans les services de santé. Non pas qu'on puisse songer à le substituer aux méthodes actuelles, plus lentes de beaucoup, plus primitives, moins perfectionnées, mais plutôt comme auxiliaire, surtout pour les secondes lignes, là où les routes sont meilleures

$11908, \mathrm{n}^{\text {os }} 13,14,15,18,19,21,22,23$. 
et où il s'agit de rejoindre le plus vite possible les voies ferrées et les trains sanitaires.

M, le Dr Dommartin passe en revue les différents genres de véhicules par traction mécạnique qui sont destinés à rendre des services pour le transport des blessés et examine dans quelles conditions peuvent y être adaptés les moyens actuels de couchage. Le choix des modèles de voitures, le mode d'aménagement de ces voitures pour le but proposé, nécessitera encore de nombreuses expériences, mais la consciencieuse étude de l'auteur, qui aborde le sujet sous toutes les faces et dans tous les détails, serre la question de très près, et, s'il ne la résout pas, du moins permet-il dores et déjà de prévoir un fonctionnement rationnel de ce service nouveau, qui, à en juger par les indications et prévisions de M. Dommartin, semble devoir prendre une importance très grande à l'avenir.

L'auteử appelle, en terminant, de ses vœux la réalisation pratique d'essais d'un service auxiliaire de santé par voitures automobiles. Sans doute ces essais ne sauraient être mieux réalisés que conformément au plan très complet tracé par lui, et seules les grandes manœuvres pourront donner une réponse quelque peu précise à cet égard. Une expérience dans ce sens ne saurait sans doute tarder à être réalisée.

$D^{r} \mathbf{F}$.

DES MOYENS DE SIGNALER AUX BLESSÉS L'EMPLACEMENT DES POSTES DE SECOURS

Cette question fait, dans le Caducée ${ }^{1}$ l'objet de deux éludes intéressantes par les docteurs de Santy et Romary. Les lecteurs du Bulletin se souviennent de la proposition faite par le $D^{r}$ de Mooj, après la guerre du Transvaal, consistant à assurer la protection des postes de secours par des signaux particuliers pour éviter toute confusion avec d'autres groupements de l'armée ennemie. De Mooj proposait pour ce but de petits ballons captifs, ou des réflecteurs spéciaux ávec croix rouge bien visible.

$1908, n^{0} 19$, p. 258 , et 21, p. 288 . 